\title{
Brownian motion with respect to a metric depending on time; definition, existence and applications to Ricci flow
}

\author{
Marc Arnaudon ${ }^{a}$, Kolehe Abdoulaye Coulibaly ${ }^{\mathrm{a}}$, Anton Thalmaier ${ }^{\mathrm{b}}$ \\ ${ }^{a}$ Laboratoire de Mathématiques et Applications (UMR6086), Université de Poitiers, Téléport 2, BP 30179, \\ 86962 Futuroscope Chasseneuil cedex, France \\ ${ }^{\mathrm{b}}$ Unité de recherche en mathématiques, Université du Luxembourg, 162A, avenue de la Faïencerie, L-1511 Luxembourg, \\ Grand-Duché de Luxembourg \\ Received 29 April 2008; accepted 6 May 2008 \\ Available online 20 June 2008 \\ Presented by Paul Malliavin
}

\begin{abstract}
Given an $n$-dimensional compact manifold $M$, endowed with a family of Riemannian metrics $g(t)$, a Brownian motion depending on the deformation of the manifold (via the family $g(t)$ of metrics) is defined. This tool enables a probabilistic view of certain geometric flows (e.g. Ricci flow, mean curvature flow). In particular, we give a martingale representation formula for a non-linear PDE over $M$, as well as a Bismut type formula for a geometric quantity which evolves under this flow. As application we present a gradient control formula for the heat equation over $(M, g(t))$ and a characterization of the Ricci flow in terms of the damped parallel transport. To cite this article: M. Arnaudon et al., C. R. Acad. Sci. Paris, Ser. I 346 (2008).

() 2008 Académie des sciences. Published by Elsevier Masson SAS. All rights reserved.

\section{Résumé}

Mouvement brownien par rapport à une famille de métriques : définition, existence et applications. Soit $M$ une variété compacte de dimension $n$ et $g(t)$ une famille de métriques sur $M$, nous allons définir un $g(t)$-mouvement brownien, qui sera l'analogue d'un mouvement brownien sur une variété mais tenant compte de la déformation (c'est-à-dire de la famille de métriques $g(t)$ ). Cet outil nous donnera une vision probabiliste de différents flots géométriques (e.g. flot de Ricci, flot de courbure moyenne). Nous donnerons aussi des formules de représentation en terme des martingales de solutions d'EDP non-linéaires sur $M$, ainsi que des formules du type Bismut pour des quantités géométriques évoluant le long d'un tel flot. Pour finir, nous donnerons comme application une formule de contrôle du gradient d'une solution de l'équation de la chaleur sur $(M, g(t))$ et une caractérisation du flot de Ricci en terme de transport parallèle déformé. Pour citer cet article: M. Arnaudon et al., C. R. Acad. Sci. Paris, Ser. I 346 (2008). ๑ 2008 Académie des sciences. Published by Elsevier Masson SAS. All rights reserved.
\end{abstract}

\section{Version française abrégée}

Soit $M$ une variété compacte de dimension $n$, munie d'une famille $C^{1}$ de métriques $g(t)$, et $\mathcal{F}(M)$ le fibré des repères sur $M$. Notons $\nabla^{g(t)}$ la connexion de Levi-Civita relative à la métrique $g(t)$ et $\Delta_{g(t)}$ l'opérateur de Laplace-

E-mail addresses: arnaudon@math.univ-poitiers.fr (M. Arnaudon), coulibal@math.univ-poitiers.fr (K.A. Coulibaly), anton.thalmaier@uni.lu (A. Thalmaier). 
Beltrami. Nous construisons une EDS de Stratonovich sur $\mathcal{F}(M)$ dont la solution en tout temps $t$ forme un système orthonormé pour $g(t)$ :

$$
\left\{\begin{array}{l}
* \mathrm{~d} \tilde{U}_{t}=L_{i}\left(t, \tilde{U}_{t}\right) * \mathrm{~d} B^{i}-\frac{1}{2}\left(\partial_{t} g\right)\left(t, \tilde{U}_{t} e_{\alpha}, \tilde{U}_{t} e_{\beta}\right) \hat{V}_{\alpha, \beta}\left(\tilde{U}_{t}\right) \mathrm{d} t, \\
\tilde{U}_{0} \in \mathcal{F}(M)
\end{array}\right.
$$

où $\left(e_{i}\right)_{i=1, \ldots, n}$ est une base orthonormée de $\mathbb{R}^{n}, L_{i}(t, u)=h^{g(t)}\left(u e_{i}\right)$ est le $\nabla^{g(t)}$ relèvement horizontal de $u e_{i}, \hat{V}_{\alpha, \beta}$ est la base canonique des champs de vecteurs verticaux de $\mathcal{F}(M)$ et $B^{i}$ sont $n$ mouvements browniens indépendants sur un espace probabilisé filtré $\left(\Omega,\left(\mathcal{F}_{t}\right), \mathbb{P}\right)$.

Définition 0.1. Soit $\left(\tilde{U}_{0} e_{i}\right)$ une base orthonormée de $\left(T_{x} M, g(0)\right)$. On définit le $g(t)$-mouvement brownien par projection : $X_{t}(x)=\pi\left(\tilde{U}_{t}\right)$.

Il peut aussi s'écrire à l'aide de développement stochastique : $* \mathrm{~d} X_{t}(x)=\tilde{U}_{t} e_{i} * \mathrm{~d} B^{i}$.

Proposition 0.2. Soit $X_{t}(x)$ un $g(t)$-mouvement brownien, alors :

$$
\left\{\begin{array}{l}
\forall f \in C^{\infty}(M), \quad \mathrm{d}\left(f\left(X_{t}(x)\right)\right) \stackrel{\mathrm{d} \mathcal{M}}{=} \frac{1}{2}\left(\Delta_{g(t)} f\right)\left(X_{t}(x)\right) \mathrm{d} t, \\
X_{0}=x .
\end{array}\right.
$$

De façon naturelle, nous définissons un transport parallèle stochastique que l'on notera :

$$
/ /_{0, t}:\left(T_{X_{0}(x)} M, g(0)\right) \longrightarrow\left(T_{X_{t}(x)} M, g(t)\right) \text {. }
$$

De telles familles de métriques sont produites naturellement par des flots géométriques (flot de courbure moyenne, flot de Ricci,...). Nous allons donner des exemples d'utilisations dans le cas du flot de Ricci et de l'équation de la chaleur associée :

$$
\left\{\begin{array}{l}
\partial_{t} f(t, x)=\frac{1}{2} \Delta_{g(t)} f(t, x), \\
\frac{\mathrm{d}}{\mathrm{d} t} g_{i j}=-\mathrm{Ric}_{i j}, \\
f(0, x)=f_{0}(x),
\end{array}\right.
$$

où $f_{0}$ est une fonction lisse sur $M$.

Le flot de Ricci sur les variétés compactes développe des singularités en temps fini. On notera $T_{c}$ le temps d'apparition de la première singularité. Pour $T<T_{c}$, on note $X_{t}^{T}(x)$ un $g(T-t)$-mouvement brownien et $/ /_{0, t}^{T}$ son transport parallèle. Notons que pour $f$ solution de (2), le processus $f\left(T-t, X_{t}^{T}(x)\right)$ est une martingale.

Définition 0.3. On définit le transport parallèle déformé $W_{0, t}^{T}: T_{x} M \rightarrow T_{X_{t}^{T}(x)} M$ comme solution de :

$$
\mathrm{d}\left(\left(/ / /_{0, t}^{T}\right)^{-1}\left(W_{0, t}^{T}\right)\right)=-\frac{1}{2}\left(/ / /_{0, t}^{T}\right)^{-1}\left(\operatorname{Ric}^{g(T-t)}-\partial_{t}(g(T-t))\right)^{\# g(T-t)}\left(W_{0, t}^{T}\right) \mathrm{d} t, \quad W_{0,0}^{T}=\mathrm{id} .
$$

Théorème 0.4. Pour tout $f_{0} \in \mathcal{F}(M)$ et $f(t, \cdot)$ solution de (2), le processus

$$
\mathrm{d} f(T-t, \cdot)_{X_{t}^{T}(x)}\left(W_{0, t}^{T} v\right), \quad 0 \leqslant t \leqslant T,
$$

est une martingale locale pour tout $v \in T_{x} M$.

Théorème 0.5. Les assertions suivantes sont équivalentes :

(i) La famille de métriques $g(t)$ évolue suivant l'équation de Ricci.

(ii) Pour tout $T<T_{c}$, on $a / /_{0, t}^{T}=W_{0, t}^{T}$.

Dans ce cas, le transport parallèle déformé est une isométrie. 


\section{Definition of a $g(t)$-BM}

Let $M$ be an $n$-dimensional manifold, $\mathcal{F}(M)$ the frame bundle, and $g(t)$ a family of metrics over $M$. Denote by $\nabla^{g(t)}$, resp. $\Delta_{g(t)}$, the associated Levi-Civita connections, resp. Laplace-Beltrami operators. Finally let $\left(e_{i}\right)_{i=1, \ldots, n}$ be an orthonormal basis of $\mathbb{R}^{n}$. For $u \in \mathcal{F}(M)$ let $L_{i}(t, u)=h^{g(t)}\left(u e_{i}\right)$ be the horizontal lift of $u e_{i}$ with respect to $\nabla^{g(t)}, L_{i}(t)$ the associated horizontal vector field, and $\hat{V}_{\alpha, \beta}$ the canonical basis of vertical vector fields. Take $B^{i}$, $i=1, \ldots, n$, independent real Brownian motions on a canonical filtered probability space $\left(\Omega,\left(\mathcal{F}_{t}\right), \mathbb{P}\right)$. Suppose that the family of metrics is $C^{1}$ in time, and consider the following Stratonovich stochastic differential equation on $\mathcal{F}(M)$ :

$$
\left\{\begin{array}{l}
* \mathrm{~d} \tilde{U}_{t}=L_{i}\left(t, \tilde{U}_{t}\right) * \mathrm{~d} B^{i}-\frac{1}{2}\left(\partial_{t} g\right)\left(t, \tilde{U}_{t} e_{\alpha}, \tilde{U}_{t} e_{\beta}\right) \hat{V}_{\alpha, \beta}\left(\tilde{U}_{t}\right) \mathrm{d} t, \\
\tilde{U}_{0} \in \mathcal{F}(M) .
\end{array}\right.
$$

Eq. (3) has local Lipschitz coefficients and hence a unique strong solution with continuous paths.

Proposition 1.1. If $\left(\tilde{U}_{0} e_{i}\right)$ is an orthonormal basis of $\left(T_{\pi\left(\tilde{U}_{0}\right)} M, g(0)\right)$ then $\left(\tilde{U}_{t} e_{i}\right)$ is an orthonormal basis of $\left(T_{\pi\left(\tilde{U}_{t}\right)} M, g(t)\right), \mathbb{P}$-a.s.

Proof. The covariant derivative determines a horizontal complement (varying as a function of time) in $T T M$ to the vertical subspace, and induces a compatible decomposition of $T \mathcal{F} M$. In the above equation, at each time the projection onto the vertical space according to the current connection $\nabla^{g(t)}$ is given. We use Schwartz principle (every construction for $C^{1}$ curves generalizes to semimartingales, by approximation of solutions of Stratonovich equations, e.g. [5], Theorem 7.24) along with a Taylor development of the metric, to compute the covariant derivative of the tangent-valued process.

For the drift part in Eq. (3) there are many choices giving the same result as Proposition 1.1. Our choice is canonical in the sense that it is symmetric and forces the solution to be a $g(t)$-isometry. If $g(t)$ does not depend on $t$, the construction reduces to the usual stochastic parallel transport, see [8].

Definition 1.2. Let $\left(\tilde{U}_{0} e_{i}\right)$ be an orthonormal basis of $\left(T_{x} M, g(0)\right)$. Define the $g(t)-\operatorname{BM}(x)$ as:

$$
X_{t}(x)=\pi\left(\tilde{U}_{t}\right) .
$$

Proposition 1.3. If $X_{t}(x)$ is a $g(t)-\operatorname{BM}(x)$ then

$$
\left\{\begin{array}{l}
\forall f \in C^{\infty}(M), \quad \mathrm{d}\left(f\left(X_{t}(x)\right)\right) \stackrel{\mathrm{d} \mathcal{M}}{=} \frac{1}{2} \Delta_{g(t)} f\left(X_{t}(x)\right) \mathrm{d} t, \\
X_{0}=x .
\end{array}\right.
$$

Proof. We compute $L_{i}(t) L_{j}(t)(f \circ \pi)(u)=\nabla^{g(t)} \mathrm{d} f\left(u e_{i}, u e_{j}\right)$ as in [6]. The result follows.

Remark 1. The formulation above gives a non-linear differential equation for the density of a $g(t)$ - $\operatorname{BM}(x)$ which is independent of the choice of the initial orthonormal frame; consequently the law of a $g(t)$ - $\operatorname{BM}(x)$ is independent of this choice. In the case where $g(t)$ is a solution of the Ricci flow the density of the $g(t)-\operatorname{BM}(x)$ satisfies an equation which is close to the conjugate heat equation.

Definition 1.4. The $g(t)$-parallel transport along the $g(t)$-BM is defined by $/ /_{0, t}=\tilde{U}_{t} \circ \tilde{U}_{0}^{-1}$. It is a linear isometry along the $g(t)-\operatorname{BM}(x) X_{t}(x)$ in the sense of isometries:

$$
/ /_{0, t}:\left(T_{X_{0}(x)} M, g(0)\right) \longrightarrow\left(T_{X_{t}(x)} M, g(t)\right) .
$$

Remark 2. Consider the mean curvature flow $\left(M_{t}\right)$ of some compact hypersurface $M_{0}$ in $\mathbb{R}^{n+1}$ as in [7], and the natural pullback of the induced metric. We obtain a family of metrics $g(t)$, and a characterization of this flow in terms of the $g(t)$-BM. For $T$ strictly smaller than explosion time, and for a $g(T-t)$-BM we have a $\mathbb{R}^{n+1}$-valued Itô martingale (with finite quadratic variation equal to $2 n t$ before explosion time) which lives in $M_{T-t}$ at time $t$ (control problem). One can derive an estimate of the explosion time in terms of the diameter of the initial hypersurface. 


\section{Damped parallel transport}

Consider

$$
\left\{\begin{array}{l}
\partial_{t} f(t, x)=\frac{1}{2} \Delta_{g(t)} f(t, x) \\
f(0, x)=f_{0}(x)
\end{array}\right.
$$

where $f_{0}$ is a smooth function on $M$ and where $\Delta_{g(t)}$ is the Laplacian on $M$ with respect to $g(t)$. Let $X_{t}^{T}(x)$ be a $g(T-t)-\operatorname{BM}(x)$ and define the $g(T-t)$-parallel transport $/ /_{0, t}^{T}$ as before.

Definition 2.1. The damped parallel transport $W_{0, t}^{T}: T_{x} M \longrightarrow T_{X_{t}^{T}(x)} M$ is defined as the solution of

$$
\mathrm{d}\left(\left(/ / /_{0, t}^{T}\right)^{-1} W_{0, t}^{T}\right)=-\frac{1}{2}\left(/ / /_{0, t}^{T}\right)^{-1}\left(\operatorname{Ric}^{g(T-t)}-\partial_{t}(g(T-t))\right)^{\# g(T-t)}\left(W_{0, t}^{T}\right) \mathrm{d} t, \quad W_{0,0}^{T}=\mathrm{id} .
$$

Theorem 2.2. For any $f_{0} \in \mathcal{F}(M)$, and $f(t, \cdot)$ a solution of (4), the process

$$
\mathrm{d} f(T-t, \cdot)_{X_{t}^{T}(x)} W_{0, t}^{T} v, \quad 0 \leqslant t \leqslant T,
$$

is a local martingale for any $v \in T_{x} M$. Here $d f$ denotes the de Rham differential of $f$.

Proof. We use scalarization of a differential form to bring computations back in $\mathbb{R}^{n}$. The proof then relies on commutativity of de Rham differential and Hodge de Rham Laplacian, along with the Weitzenböck formula for differential forms.

Taking $\frac{\mathrm{d}}{\mathrm{d} t} g_{i j}(t)=-\mathrm{Ric}_{i j}(t)$ as definition of the forward Ricci flow, the associated heat equation is:

$$
\left\{\begin{array}{l}
\partial_{t} f(t, x)=\frac{1}{2} \Delta_{g(t)} f(t, x), \\
\frac{\mathrm{d}}{\mathrm{d} t} g_{i j}=-\mathrm{Ric}_{i j}, \\
f(0, x)=f_{0}(x)
\end{array}\right.
$$

where $f_{0}$ is a smooth function on $M$.

Remark 3. The Ricci flow of a compact manifold develops its first singularity in finite time which we denote by $T_{c}$. For $T<T_{c}$ we take $X_{t}^{T}(x)$ as a $g(T-t) \operatorname{BM}(x)$, therefore if $f(t, x)$ is a solution of (5) then $f\left(T-t, X_{t}^{T}(x)\right)$ is a martingale. Eq. (3) then becomes

$$
\left\{\begin{array}{l}
* \mathrm{~d} U_{t}^{T}=L_{i}\left(T-t, U_{t}^{T}\right) * \mathrm{~d} B^{i}-\frac{1}{2} \operatorname{Ric}^{g(T-t)}\left(U_{t}^{T} e_{\alpha}, U_{t}^{T} e_{\beta}\right) \hat{V}_{\alpha, \beta}\left(U_{t}^{T}\right) \mathrm{d} t \\
U_{0}^{T} \text { a } g(T) \text {-orthonormal basis of } T_{x} M
\end{array}\right.
$$

We denote by $/ /_{0, t}^{T}$ again the $g(T-t)$-parallel transport.

Remark 4. There is an analogous result for the backward Ricci flow and its heat flow which are defined as before but with a sign change in front of the Ricci curvature in the evolution of the metric.

Theorem 2.3. Keeping the notions from above, the following items are equivalent:

(i) A family $g(t)$ of metrics evolves under the forward Ricci flow.

(ii) For any $T<T_{c}$, the equality $/ /_{0, t}^{T}=W_{0, t}^{T}$ holds.

In this case the damped parallel transport is an isometry. 
Remark. With respect to a fixed metric, the damped parallel transport differs in general from the usual parallel transport. In our case the evolution of the metric under the forward Ricci flow cancels the damping of the parallel transport. A nice consequence for computations is that in this case the damped parallel transport is an isometry. This is not the case for the backward Ricci flow.

\section{Bismut formula for the Ricci flow}

We intend to give a Bismut type gradient formula for the solution of Eq. (5), depending only on the initial condition $\left(f_{0}, g(0)\right)$, see $[3,4,9,1]$. To this end, we derive the following integration by parts formula:

Lemma 3.1. For any $\mathbb{R}^{n}$-valued process $k$ in $L_{\text {loc }}^{2}(B)$ where $B$ is an $\mathbb{R}^{n}$-valued Brownian motion,

$$
N_{t}=\mathrm{d} f(T-t, \cdot)_{X_{t}^{T}(x)}\left(U_{t}^{T}\right)\left[\left(U_{0}^{T}\right)^{-1} v-\int_{0}^{t} k_{r} \mathrm{~d} r\right]+f\left(T-t, X_{t}^{T}(x)\right) \int_{0}^{t}\left\langle k_{r}, \mathrm{~d} B_{r}\right\rangle_{\mathbb{R}^{n}}
$$

is a local martingale.

Proof. By Proposition 1.3, $f\left(T-t, X_{t}^{T}(x)\right)$ is a martingale and its Itô differential is:

$$
d\left(f\left(T-t, X_{t}^{T}(x)\right)\right)=\mathrm{d} f(T-t, \cdot)_{X_{t}^{T}(x)} U_{t}^{T}\left(U_{0}^{T}\right)^{-1} e_{i} \mathrm{~d} B^{i},
$$

where $\left(e_{i}\right)_{i=1, \ldots, n}$ is a $g(T)$-orthonormal basis of $T_{x} M, \mathrm{~d} B=\left(U_{0}^{T}\right)^{-1} e_{i} \mathrm{~d} B^{i}$. With Theorem 2.2 we get:

$$
\begin{aligned}
\mathrm{d} N_{t} \stackrel{\mathrm{d} \mathcal{M}}{=} & -\sum_{i} \mathrm{~d} f(T-t, \cdot)_{X_{t}^{T}(x)} U_{t}^{T}\left(U_{0}^{T}\right)^{-1} e_{i}\left\langle U_{0}^{T}\left(k_{t}\right), e_{i}\right\rangle_{T} \mathrm{~d} t+\mathrm{d}\left(f\left(T-t, X_{t}^{T}(x)\right)\right)\left\langle k_{t}, \mathrm{~d} B\right\rangle_{\mathbb{R}^{n}} \\
\stackrel{\mathrm{d} \mathcal{M}}{=} & -\sum_{i} \mathrm{~d} f(T-t, \cdot)_{X_{t}^{T}(x)} U_{t}^{T}\left(U_{0}^{T}\right)^{-1} e_{i}\left\langle U_{0}^{T}\left(k_{t}\right), e_{i}\right\rangle_{T} \mathrm{~d} t \\
& \quad+\mathrm{d} f(T-t, \cdot)_{X_{t}^{T}(x)}\left(\sum_{i} U_{t}^{T}\left(U_{0}^{T}\right)^{-1} e_{i} \mathrm{~d} B^{i}\right)\left(\sum_{j}\left\langle k_{t},\left(U_{0}^{T}\right)^{-1} e_{j}\right\rangle \mathrm{d} B^{j}\right) \\
& \stackrel{\mathrm{d} \mathcal{M}}{=} 0,
\end{aligned}
$$

which proves the lemma.

We can immediately deduce that the supremum of the gradient in the space variable of a solution to Eq. (5) decreases in time with a bound specified in the following corollary.

Corollary 3.2. Let $\mathcal{M}_{0}=\sup _{M}\left|f_{0}\right|$. For $T<T_{c}$ we have:

$$
\sup _{x \in M}\left\|\nabla^{T} f(T, x)\right\|_{T} \text { is decreasing in time, and } \sup _{x \in M}\left\|\nabla^{T} f(T, x)\right\|_{T} \leqslant \frac{\mathcal{M}_{0}}{\sqrt{T}} .
$$

Proof. Take $x \in M$ such that $\left\|\nabla^{T} f(T, x)\right\|_{T}$ is maximal. The first result follows from Theorems 2.2 and 2.3. Choose $k_{r}=\left(U_{0}^{T}\right)^{-1} v / T \mathbf{1}_{[0, T]}$ in Lemma 3.1, then $N_{t}$ is a martingale; taking expectation at time 0 and $T$, we get

$$
\mathrm{d} f(T, \cdot)_{x} v=\frac{1}{T} \mathbb{E}\left[f_{0}\left(X_{T}^{T}(x)\right) \int_{0}^{T}\left\langle\left(U_{0}^{T}\right)^{-1} v, \mathrm{~d} B\right\rangle_{\mathbb{R}^{n}}\right] .
$$

Given $x \in M$ and $v=\nabla^{T} f(T, x)$, Schwartz inequality gives

$$
\left\|\nabla^{T} f(T, x)\right\|_{T}^{2} \leqslant \frac{\mathcal{M}_{0}}{T} \mathbb{E}\left[\left|\int_{0}^{T}\left\langle\left(U_{0}^{T}\right)^{-1} v, \mathrm{~d} W\right\rangle_{\mathbb{R}^{n}}\right|^{2}\right]^{1 / 2} .
$$


The result follows.

In the case of the normalized Ricci flow of surfaces (which is well understood), we can derive an estimate for the gradient of the scalar curvature. Let $M$ be 2-dimensional, $R(t)$ the scalar curvature, and $r:=\int_{M} R(t) \mathrm{dvol} / \int_{M} \mathrm{dvol}$ its average (constant in time). We consider the normalized Ricci flow (which conserves the volume), i.e.,

$$
\frac{\mathrm{d}}{\mathrm{d} t} g_{i j}(t)=(r-R(t)) g_{i j}(t) .
$$

Remark 6. Hamilton (see [2]) gives a proof of all time existence of solutions to this equation. He also establishes a result of convergence of such metrics to a metric of constant curvature.

Proposition 3.3. For $T>0$, let $X_{t}^{T}(x)$ be a $\frac{1}{2} g(T-t)-\mathrm{BM}(x)$ and $/ /_{0, t}^{T}$ be the parallel transport along $X_{t}^{T}(x)$. Further, for $v \in T_{x} M$, let $\phi_{t} v$ be the solution to the following Itô equation:

$$
/ /{ }_{0, t}^{T} \mathrm{~d}\left(\left(/ /{ }_{0, t}^{T}\right)^{-1} \phi_{t} v\right)=-\left(3 r / 2-2 R\left(T-t, X_{t}^{T}(x)\right)\right) \phi_{t} v \mathrm{~d} t .
$$

Then $\mathrm{d} R(T-t, \cdot)_{X_{t}^{T}(x)} \phi_{t} v$ is a martingale, and the following estimate holds:

$$
\left\|\nabla^{T} R(T, x)\right\|_{T} \leqslant \sup _{M}\left\|\nabla^{0} R(0, \cdot)\right\|_{0} \mathrm{e}^{-3 r T / 2} \mathbb{E}\left[\exp \left(\int_{0}^{T} 2 R\left(T-t, X_{t}^{T}(x)\right) \mathrm{d} t\right)\right] .
$$

Remark 7. In the case $\chi(M)<0$ (which is the easiest case) we can give an estimate which decreases exponentially in time, using Prop. 5.18 in [2]. In this case we have $r<0$, a constant $C$ depending only on the initial metric, and the following estimate for all time:

$$
\left\|\nabla^{T} R(T, x)\right\|_{T} \leqslant \sup _{M}\left\|\nabla^{0} R(0, \cdot)\right\|_{0} \mathrm{e}^{r T / 2} \exp \left(2 C \frac{\mathrm{e}^{r T}-1}{r}\right) .
$$

In Proposition 3.3, we were interested in the $\mathrm{e}^{-3 r T / 2}$ term which may, in the case $\chi(M)>0$, give convergence of the gradient of scalar curvature of a surface evolving by normalized Ricci flow to 0 . For such a conclusion it is sufficient to estimate the expectation term in (6).

\section{References}

[1] M. Arnaudon, B.K. Driver, A. Thalmaier, Gradient estimates for positive harmonic functions by stochastic analysis, Stochastic Process Appl. 117 (2) (2007) 202-220.

[2] B. Chow, D. Knopf, The Ricci Flow: An Introduction, Mathematical Surveys and Monographs, vol. 110, American Mathematical Society, Providence, RI, 2004.

[3] K.D. Elworthy, X.-M. Li, Formulae for the derivatives of heat semigroups, J. Funct. Anal. 125 (1) (1994) 252-286.

[4] K.D. Elworthy, M. Yor, Conditional expectations for derivatives of certain stochastic flows, in: Séminaire de Probabilités, XXVII, in: Lecture Notes in Math., vol. 1557, Springer, Berlin, 1993, pp. 159-172.

[5] M. Émery, Stochastic Calculus in Manifolds, Springer-Verlag, Berlin, 1989. With an appendix by P.-A. Meyer.

[6] E.P. Hsu, Stochastic Analysis on Manifolds, Graduate Studies in Mathematics, vol. 38, American Mathematical Society, Providence, RI, 2002.

[7] G. Huisken, Flow by mean curvature of convex surfaces into spheres, J. Differential Geom. 20 (1) (1984) 237-266.

[8] P. Malliavin, Stochastic Analysis, Grundlehren der Mathematischen Wissenschaften, vol. 313, Springer, Berlin, 1997.

[9] A. Thalmaier, Feng-Yu Wang, Gradient estimates for harmonic functions on regular domains in Riemannian manifolds, J. Funct. Anal. 155 (1) (1998) 109-124. 